\title{
3D reconstruction of large scale city models as a support to Sustainable Development
}

\author{
Jean-Philippe Pons ${ }^{1}$ and Souheil Soubra ${ }^{2}$ \\ ${ }^{1}$ CERTIS, École des ponts, Université Paris-Est, Marne-la-Vallée, France \\ ${ }^{2}$ MOD-EVE, Centre Scientifique et Technique du Bâtiment, Sophia-Antipolis, France \\ souheil.soubra@cstb.fr
}

\begin{abstract}
No part of the economic community can now escape from the urgent issues related to global warming, carbon footprint and reducing energy consumption. Nevertheless, the construction sector is particularly under pressure. Indeed, it is one of the biggest consumers of energy. It also largely contributes to the massive use of some critical resources (such as energy, water, materials and space...) and is responsible for a large portion of greenhouse gas emissions. In that context, the paper explores new approaches for urban planning by combining Virtual Environments and Simulations to address sustainability issues. These approaches are based on the possibilities of reconstructing 3D models of the built environment using standard photographs taken with off-the shelf handheld digital cameras. The 3D models can then be combined with simulations in order to address sustainable urban development issues.
\end{abstract}

Keywords: Total life cycle support, 3D virtual world environments

\section{Introduction}

Cities are dynamic living organisms that are constantly evolving, which makes city planning a very complex process involving a large number of actors: planners, developers, communities, environmental groups, local and national governmental agencies, civil security organisations, etc. Each of them represents their own interests, the interests of an organisation or the public interest. Handling this complex process is essential since it has a direct impact on the quality of life of all citizens and the economic, social and governance manifestations of their citizenship.

Currently, the complexity of the city planning process is amplified by the overwhelming number of challenges facing the society. Among these challenges, global warming and the end of low cost fossil resources are of paramount importance.

From the technological point of view, there is a genuine need for innovative design and urban planning tools that enhance creativity and allow to investigate "out of the box" solutions that are now abandoned due to their complexity in order to tackle these complex interdisciplinary issues. We consider that this emerging generation of tools will integrate various dimensions such as linking with geographical information, constructing high quality 3D models of the built environment and using numerical models and simulation tools in order to play "what-if" scenarios and predict the impact of the proposed design and planning options. 


\section{Methods}

The problem of automatic 3D object/scene modeling from digital photographs has received a lot of attention in the computer vision/graphics communities, as a potential replacement for laser range scanning, advantageously cheaper and more flexible. However, until recently, existing techniques have been limited to particular setups (turntable), simple scenes (single compact object), and/or unrealistic imaging conditions (controlled lighting, simple or known background). Also, they did not scale well with the number of images and scene complexity. Consequently, they were not applicable to real-world large-scale natural or architectural scenes.

Recent progress, fostered by a few international research teams, including ours, has led to reconsider the above statements, and has allowed us to design a prototype system for automatic image-based 3D modeling.

The input to our system is a sequence of photographs of a scene taken with a standard hand-held digital camera. Note that $3 \mathrm{D}$ reconstruction is possible only for parts of the scene photographed from at least two viewpoints. This leads to regularly changing the camera position and leaving significant overlap while taking pictures. The output of our system is a textured 3D model compatible with standard 3D viewers. Our system follows an algorithmic procedure roughly composed of three steps, which we describe in the next subsections.

\subsection{Camera self-calibration}

The first step of the approach consists in automatically estimating the position and the orientation of each shot, as well as associated internal camera parameters, such as focal length or radial distortion. This difficult task, known as camera self-calibration in the computer vision area, has been extensively studied for the last two decades. However, it may still be the bottleneck when the number of images exceeds one hundred. Following [1], we first estimate pair-wise relative rotations and translations by robustly matching, between some image pairs, a few key points [2] corresponding to the same material scene points. Globally optimal camera poses can then be efficiently computed through a matrix eigenvalue decomposition.

\subsection{Geometry estimation}

We have developed novel methods to automatically, accurately and robustly reconstruct the 3D shape of a scene from several calibrated images. Our method [3] first generates a quasi-dense point cloud of the scene by matching keypoints in a lenient manner, thus possibly retaining many false matches. Second, it builds an adaptive tetrahedral decomposition of space by computing the 3D Delaunay triangulation of the 3D point set. Third, it reconstructs the scene by labeling Delaunay tetrahedra as empty or occupied, thus generating a triangular mesh of the scene. A globally optimal label assignment is efficiently found as a minimum cut solution in a graph. Finally, the obtained model is refined with a deformable mesh [4] by local minimization of some multi-view consistency score [5]. 


\subsection{Visual attributes estimation}

In this step, we estimate the visual attributes of the 3D model by assembling the redundant color information of the input images into a single rectangular texture map, known as a texture atlas: it allows us to output a portable and efficiently renderable 3D format. Our algorithm [6] focuses on avoiding visual artifacts such as color discontinuities, ghosting or blurring, which typically arise from photometric and geometric inaccuracies: varying light conditions and camera response, highlights, imperfect camera calibration, approximate shape, etc. To do so, we compute a partition of the 3D surface which realizes a good trade-off between visual detail and color continuity at patch boundaries. We then apply a pixel-wise color correction in the vicinity of patch boundaries, adaptively for each image frequency band, in order to achieve faultless color continuity while avoiding ghosting artifacts.

\section{Results and discussion}

In this section, we demonstrate our image-based 3D modeling system on two challenging real-world datasets:

- “Aiguille du Midi": 37 images of a famous French peak (Chamonix Mont-Blanc), copyright Bernard Vallet (www.bvallet.com).

- "Ettlingen Castle": 19 images of Ettlingen Castle (Germany), courtesy Christoph Strecha, EPFL.

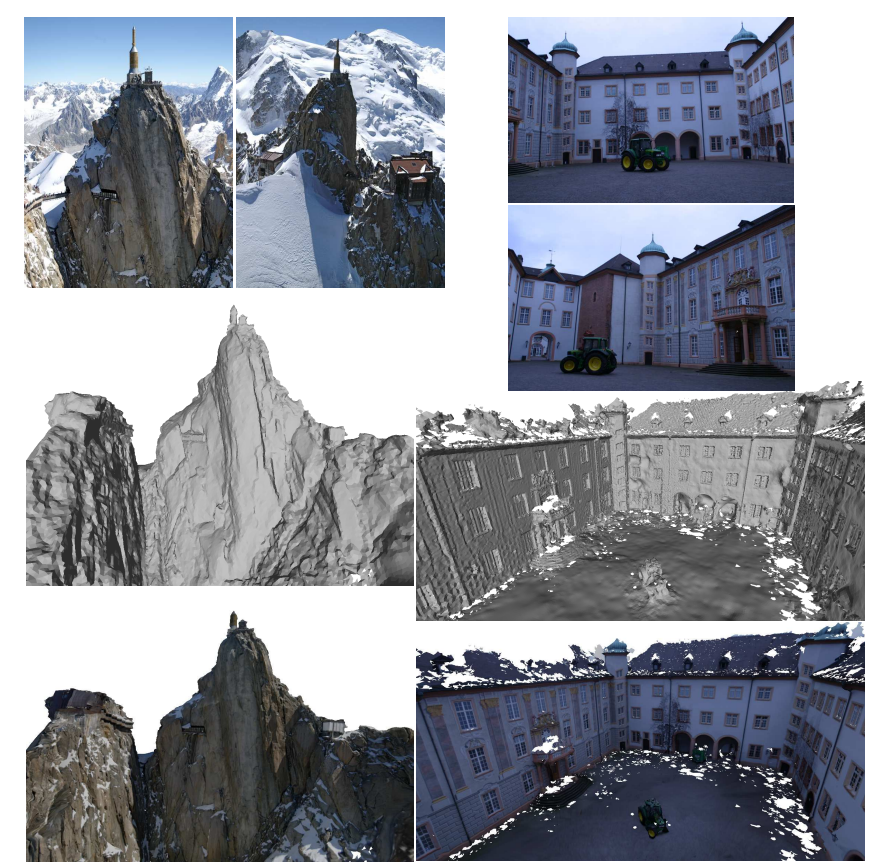

Fig. 1. First results on two challenging real-world datasets. See text for details. 
For each dataset, Figure 1 displays two sample input images (top row), a view of the estimated geometric model (middle row) and a view of the full model (bottom row). Note how the level of geometric detail and the photo-realism significantly surpasses conventional CAD techniques (see http://certis.enpc.fr for more details).

\section{Conclusion}

Pressure for sustainable development is increasing rapidly in all industrial sectors due to soaring oil prices and concerns about climate change. This is particularly true for the construction sector which is one of the biggest consumers of energy and responsible for a large portion of greenhouse gas emissions. In order to address sustainable construction in a convincing way, it is of paramount importance to evaluate the construction project as a whole taking into account, in the same process, the architectural dimension, the climate, the integration in the site, the envelope and the equipments. All approaches tending to decompose these elements and address them individually will yield inacceptable additional costs and insufficient results [7]. Currently, most of the studies tend to represent the buildings as separate entities and neglect the importance of the interactions between the building and the urban scale. One of the reasons for this is the difficulty of modelling complex urban geometry. The paper presented a state of the art method allowing to produce large scale, high quality $3 \mathrm{D}$ models of the built environment at acceptable costs. This will put 3D models of the projects at the disposal of local authorities, architects and planners for public presentations. The $3 \mathrm{D}$ model can then be used to provide input data to simulation tools in order to assess various sustainability aspects (e.g. internal air quality, thermal comfort and energy consumption, noise and air pollution).

\section{References}

1. Martinec, D., Pajdla, T.: Robust rotation and translation estimation in multiview reconstruction. In IEEE Conf. on Computer Vision and Pattern Recognition, 2007.

2. Lowe, D.: Distinctive image features from scale-invariant keypoints. The Int. J. of Computer Vision, 20, pp. 91-110, 2003.

3. Labatut, P., Pons, J.-P., Keriven, R.: Efficient multi-view reconstruction of large-scale scenes using interest points, Delaunay triangulation and graph cuts. In IEEE Int. Conf. on Computer Vision, 2007.

4. Pons, J.-P., Boissonnat, J.-D.: Delaunay deformable models: Topology-adaptive meshes based on the restricted Delaunay triangulation. In IEEE Conf. on Computer Vision, 2007.

5. Pons, J.-P., Keriven, R., Faugeras, O.: Multi-view stereo reconstruction and scene flow estimation with a global image-based matching score. The Int. J. of Computer Vision, 72(2), pp. 179-193, 2007.

6. Allène, C., Pons, J.-P., Keriven, R.: Seamless image-based texture atlases using multi-band blending. Submitted to Int. Conf. on Pattern Recognition, 2008.

7. Soubra, S.: The need for creativity enhancing design tools. In: Virtual Futures for Design, Construction \& Procurement. Peter Brandon (Eds). Blackwell Publishing (2008). 